\title{
HIERARCHICAL HYBRID MLP/HMM OR RATHER MLP FEATURES FOR A DISCRIMINATIVELY TRAINED GAUSSIAN HMM: A COMPARISON FOR OFFLINE HANDWRITING RECOGNITION
}

\author{
Philippe Dreuw, Patrick Doetsch, Christian Plahl, and Hermann Ney \\ Human Language Technology and Pattern Recognition \\ Computer Science Department \\ RWTH Aachen University, D-52056 Aachen, Germany
}

\begin{abstract}
We use neural network based features extracted by a hierarchical multilayer-perceptron (MLP) network either in a hybrid MLP/HMM approach or to discriminatively retrain a Gaussian hidden Markov model (GHMM) system in a tandem approach.

MLP networks have been successfully used to model long-term and non-linear features dependencies in automatic speech and optical character recognition. In offline handwriting recognition, MLPs have been mostly used for isolated character and word recognition in hybrid approaches. Here we analyze MLPs within an LVCSR framework for continuous handwriting recognition using discriminative MMI/MPE training. Especially hybrid MLP/HMM and discriminatively retrained MLP-GHMM tandem approaches are evaluated.

Significant improvements and competitive results are reported for a closed-vocabulary task on the IfN/ENIT Arabic handwriting database and for a large-vocabulary task using the IAM English handwriting database.
\end{abstract}

\section{INTRODUCTION}

State-of-the-art speech recognition systems are based on discriminative Gaussian hidden Markov models (GHMMs), where major points of criticism of this conventional approach are the indirect parameterization of the posterior model, the non-convexity of the conventional training criteria, and the insufficient flexibility of the HMMs to incorporate additional dependencies and knowledge sources [9].

In off-line handwriting recognition, the authors of [2] apply the Minimum Classification Error (MCE) criterion to the problem of recognizing online unconstrained-style characters and words, and report large improvements on a writerindependent character recognition task when compared to a maximum likelihood (ML) trained baseline system.

In $[4,5]$ the authors apply a margin-based Maximum $\mathrm{Mu}$ tual Information (M-MMI) and Minimum Phone Error (MMPE) training criterion to an HMM-based off-line handwriting recognition system and report up to 33\% relative improve- ment in word error rate (WER) compared to an ML trained baseline system.

State-of-the-art handwritten text recognition system are usually based on GHMMs too [1], with hybrid neural network [7, 8] or tandem based approaches [16] being recently very successful in online and off-line handwriting recognition.

However, most of the tandem based approaches use an ML based training criterion to retrain the GHMMs. In this work, we therefore investigate in and contribute to the following topics:

- We directly compare hybrid and tandem based approaches

- We analyze the alignment quality impact in MLP training

- We analyze discriminative MLP features for discriminatively trained GHMMs

- We evaluate on isolated word and large-vocabulary continuous handwriting recognition tasks

In the following we describe how we combine both approaches, i.e. discriminative GHMMs using discriminative MLP based features. We make use of our novel RWTH OCR system $^{1}$, which is based on a large vocabulary continuous speech recognition (LVCSR) framework known as RWTH $\mathrm{ASR}^{2}$. Both systems are publicly available.

\section{MLP BASED FEATURE EXTRACTION}

Without any preprocessing of the input images, we extract simple appearance-based image slice features $x_{t}$ at every time step $t=1, \cdots, T$ which are augmented by their temporal derivatives in horizontal direction $\Delta=x_{t}-x_{t-1}$.

These augmented raw slice features $X_{t}=\left[x_{t}, \Delta\right]$ together with their corresponding state alignments are then processed by a hierarchical MLP framework originally described in [17]. Depending on the MLP hierarchy, preprocessing, and postprocessing operations, several feature sets can be generated. In order to incorporate temporal and spatial context into the features, we concatenate consecutive features in a sliding

\footnotetext{
1http://www.hltpr.rwth-aachen. de/rwth-ocr/

${ }^{2}$ http://www.hltpr.rwth-aachen. de/rwth-asr/
} 


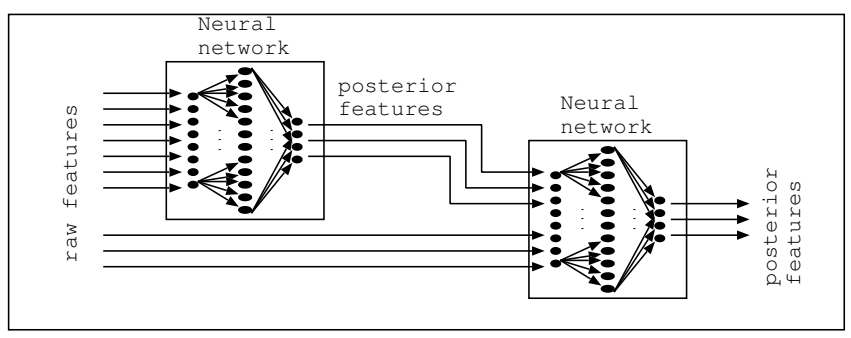

Fig. 1. Hierarchical MLP network for feature extraction

window, where the MLP outputs are later reduced by a PCA or LDA transformation (cf. Figure 1). Two different MLPs are trained, RAW and TRAP-DCT networks, where network details are given in section 4 .

Instead of using log-PCA/LDA reduced MLP posterior features for retraining a Gaussian HMM system, log-posterior features can be directly used without any reduction in a hybrid MLP/HMM framework [3], as briefly described in section 3 .

\section{HMM BASED HANDWRITING RECOGNITION}

In offline handwriting recognition, we are searching for an unknown word sequence $W_{1}^{N}:=W_{1}, \ldots, W_{N}$, for which the sequence of features $X_{1}^{T}:=X_{1}, \ldots, X_{T}$ fits best to the trained models. We maximize the posterior probability $p\left(W_{1}^{N} \mid X_{1}^{T}\right)$ over all possible word sequences $W_{1}^{N}$ with unknown number of words $N$. This is modeled by Bayes' decision rule:

$$
X_{1}^{T} \rightarrow \hat{W}_{1}^{N}\left(X_{1}^{T}\right)=\arg \max _{W_{1}^{N}}\left\{p^{\kappa}\left(W_{1}^{N}\right) p\left(X_{1}^{T} \mid W_{1}^{N}\right)\right\}
$$

with $\kappa$ being a scaling exponent of the language model.

GHMM. Our baseline system is a hidden Markov model (HMM) system which is Viterbi trained using the maximumlikelihood (ML) training criterion. As features we directly use the raw slice features within a sliding window of size 7 which are reduced by PCA to 30 components.

The ML trained Gaussian mixture models (GMMs) are retrained using a discriminative training approach based on the margin-based M-MMI/M-MPE criteria as presented in [11].

Hybrid MLP/HMM. The MLP posterior probabilities $p(S \mid X)$ are divided by the prior state probabilities $p(S)$ in order to approximate the observation probabilities of an HMM, i.e. $p(X \mid S) \approx \frac{p(S \mid X)}{p(S)}$ as described in [3], i.e. $-\log p(X \mid S)=$ $-\log p(S \mid X)+\alpha \log p(S)$ with $\alpha$ being a priori scaling factor in our log-linear framework ${ }^{3}$.

MLP-GHMM. The tandem MLP-GHMM is retrained from scratch using the MLP log-posterior features as described in section 2 (also cf. [16]). Again, different training criteria [10] can be used. Note that the MLP network itself can also be initialized using the different GHMM baseline system's alignments (cf. alignment impact in Table 3).

\footnotetext{
${ }^{3}$ a tuning of the priori scaling factor $\alpha$ lead to no improvements
}

\section{EXPERIMENTAL RESULTS}

\subsection{Isolated Word Recognition}

The IfN/ENIT database is divided into four training folds with an additional fold for testing. The current database version (v2.0p1e) contains a total of 32492 Arabic words handwritten by about 1000 writers, and has a vocabulary size of 937 Tunisian town names. Here, we follow the same evaluation protocol as for the ICDAR and ICFHR Arabic handwriting recognition competitions [14]. Due to a character and position dependent length modeling of the 28 base Arabic characters [6], we finally model the Arabic words by 216 different character labels. The system described in [4] is used to generate an initial alignment of the features to the 216 labels. Our GHMM baseline system (see Table 1) uses 3 mixtures per character label, resulting in up to 646 mixtures with $55 \mathrm{k}$ densities. The MLP networks have been trained on raw pixel column features from the sets $a, b$, and $c$ only.

RAW MLP Features. The hierarchical system uses at the first level no windowing of the input features, a single hidden layer with 2000 nodes, and 216 output nodes, which are reduced by a log-PCA transformation to 32 components. The second network concatenates these features in addition to the raw features, and uses a window size of 9 consecutive features The 576-dimensional features (i.e. $32 \times 2 \times 9$ features) are forwarded to a single hidden layer with 3000 nodes, and reduced by a log-PCA transformation to 32 components.

TRAP-DCT MLP Features. The system uses a TRAP-DCT [12] preprocessing of the raw pixel input features. The hierarchical system uses at the first level a spatio-temporal TRAPDCT window to augment the 32-dimensional raw pixel input feature vectors to a 256-dimensional vector. Again, the first level hierarchical network uses a single hidden layer with 1500 nodes, and 216 output nodes, which are reduced by a log-LDA transformation to 96 components. The second network concatenates these features in addition to the raw features, and uses a window size of 5 consecutive log-LDA network features, and a window size of 9 consecutive raw input features to account for different spatio-temporal information. The 768-dimensional features (i.e. $96 \times 5+32 \times 9$ features) are forwarded to a single hidden layer with 3000 nodes, and reduced by a log-LDA transformation to 36 components.

We empirically optimized different MLP feature combinations on the IfN/ENIT training folds, which showed no significant difference. The TRAP-DCT log-posterior features are used in Table 1 for the hybrid MLP/HMM approach, which turned out to perform slightly better than the RAW features in these informal experiments. Furthermore, we observed that a discriminative MLP-GHMM system is about $25 \%$ relative better than a generatively trained one, especially in combination with concatenated RAW+TRAP-DCT features. The comparison in Table 1 shows a significant advantage of the retrained tandem MLP-GHMM system over the hybrid MLP/HMM and the GHMM baseline. 
Table 1. System comparison: MLP-GHMM performs best, both GHMM and MLP-GHMM systems are M-MMI trained (WER/CER in [\%])

\begin{tabular}{|c|c|c|c|c|c|c|c|}
\hline \multirow[t]{2}{*}{ Train } & \multirow[t]{2}{*}{ Test } & \multicolumn{2}{|c|}{ GHMM } & \multicolumn{2}{|c|}{ MLP/HMM } & \multicolumn{2}{|c|}{ MLP-GHMN } \\
\hline & & WER & CER & WER & CER & WER & CEF \\
\hline $\mathrm{cc}$ & d & 6.1 & 2.4 & 4.5 & 1.7 & 3.5 & \\
\hline d & $\mathrm{c}$ & 6.8 & & 2.6 & 0.9 & 1. & \\
\hline$d$ & b & 6.1 & 2.2 & 2.7 & 0.9 & 2.5 & \\
\hline bcd & $\mathrm{a}$ & 7.0 & 3.1 & 3.1 & 1.3 & 2.6 & \\
\hline abcd & $\mathrm{e}$ & 15.4 & 6.1 & 11.6 & 4.5 & 7.3 & \\
\hline
\end{tabular}

Table 2. Comparison to ICDAR / ICFHR Arabic handwriting recognition competition results on IfN/ENIT

\begin{tabular}{|c|c|c|c|c|}
\hline \multirow[t]{2}{*}{ Competition / Group } & \multicolumn{4}{|c|}{ WER [\%] } \\
\hline & abc-d & abcd-e & abcde-f & abcde-s \\
\hline \multicolumn{5}{|l|}{ ICDAR 2009 [13] } \\
\hline MDLSTM (RNN/CTC) & - & - & 6.7 & 18.9 \\
\hline $\mathrm{A} 2 \mathrm{iA}(\mathrm{GHMM})$ & - & - & 17.8 & 33.6 \\
\hline A2iA (ANN/HMM) & - & - & 14.5 & 29.6 \\
\hline \multicolumn{5}{|l|}{ ICFHR 2010 (this work) } \\
\hline GHMM [4] & 6.0 & 14.6 & 14.3 & 27.5 \\
\hline MLP-GHMM (S & 3.5 & 7.3 & 9.1 & 18.9 \\
\hline MLP-GHMM (Sys.-2) & 3.7 & 7.6 & 9.1 & 19.7 \\
\hline
\end{tabular}

ICFHR Arabic handwriting recognition competition. In the following we briefly describe our HMM systems submitted to the ICFHR Arabic handwriting recognition competition [14]. The MLP network has been trained for both systems with an alignment generated by the ML trained GHMM baseline system. The submitted System-1 is a discriminatively trained system using the M-MMI criterion [11] and the 68-dimensional concatenated MLP features. System-2 is an ML trained MLP-GHMM baseline system as described in [6], which uses 8 splits with up to 256 densities per mixture and 3 mixtures per character label, resulting in about $82 \mathrm{k}$ densities.

In Table 2 it can be seen that both our GHMM and MLPGHMM systems outperform the hybrid system of the A2iA group. Furthermore it can be observed that the performance of our MLP-GHMM is close to the performance of the hybrid RNN/CTC system: as the CTC framework is very similar to an HMM based decoding, the main power probably comes from the tuned recurrent neural network (RNN).

\subsection{Large-Vocabulary Recognition}

Experiments for continuous sentence recognition are conducted on the IAM database [15] using a large-vocabulary lexicon and additional external language model resources as proposed in [1]. It contains a total number of 1,539 pages with 5,685 sentences in 9,862 lines. All words are build using only 79 different symbols which consist of both upper- and
Table 3. Alignment-impact analysis in MLP training on IAM (Note: MLP-GHMMs are ML trained here)

\begin{tabular}{|c|c|c|c|c|}
\hline \multirow[t]{2}{*}{ Systems } & \multicolumn{2}{|c|}{ WER [\%] } & \multicolumn{2}{|c|}{ CER [\%] } \\
\hline & Devel & Eval & Devel & Eval \\
\hline GHMM, ML baseline & 31.9 & 38.9 & 8.4 & 11.7 \\
\hline GHMM, M-MPE baseline & 24.3 & 30.0 & 6.8 & 10.9 \\
\hline \multicolumn{5}{|l|}{ MLP/HMM, RAW } \\
\hline + ML alignment for MLP & 37.2 & 43.3 & 12.9 & 17.4 \\
\hline + M-MPE alignment for MLP & 34.3 & 40.4 & 11.8 & 16.3 \\
\hline \multicolumn{5}{|l|}{ MLP/HMM, TRAP-DCT } \\
\hline + ML alignment for MLP & 32.7 & 39.5 & 11.0 & 15.9 \\
\hline + M-MPE alignment for MLP & 31.2 & 36.9 & 10.0 & 14.2 \\
\hline \multicolumn{5}{|l|}{ MLP-GHMM, Raw } \\
\hline + ML alignment for MLP & 27.1 & 34.3 & 8.3 & 13.2 \\
\hline + M-MPE alignment for MLP & 25.7 & 32.9 & 7.7 & 12.4 \\
\hline \multicolumn{5}{|l|}{ MLP-GHMM, TRAP-DCT } \\
\hline + ML alignment for MLP & 29.0 & 36.8 & 9.8 & 15.3 \\
\hline + M-MPE alignment for MLP & 26.7 & 33.7 & 8.4 & 13.3 \\
\hline
\end{tabular}

lowercase characters, punctuation, quotation marks, a special symbol for crossed out words, and a white-space model. We use a Kneser-Ney smoothed trigram LM with $\kappa=25.0$ (cf. Equation 1) and a 50k lexicon. Each character in our ML trained GHMM baseline system is modeled by a 10 -state left-to-right HMM with five separate GMMs, resulting in 391 mixtures with 25k Gaussian densities after ML training and globally pooled diagonal variances [5]. The MLP networks are similar to the IfN/ENIT networks, except that we use 80 output nodes in each hierarchy layer. For the TRAP-DCT MLP networks we use a log-LDA reduction to 40 components in the first and second network.

In Table 3 we keep all tuning parameters fixed and analyze the impact of ML/M-MMI/M-MPE state alignments in MLP training. As expected the quality of initial feature labeling in MLP training has a significant impact, as the M-MPE alignments always outperform the ML alignments. Note that an alternating optimization of MLP and MLP-GHMM systems via realignments did so far not further improve the overall performance. Furthermore it can be observed that MLP features help in general, and that RAW MLP features seem to outperform TRAP-DCT features.

A comparison of our proposed framework to other approaches is given in Table 4, where a strong improvement by M-MPE training in combination with RAW MLP features can be observed.

\section{CONCLUSIONS}

Significant improvements could be achieved by means of MLP based features for an HMM-based handwriting recognition system. Due to the nature of the chosen framework and databases, it can be assumed that most results can be 
Table 4. Evaluation of the proposed MLP-GHMM framework on the IAM database

\begin{tabular}{|c|c|c|c|c|}
\hline \multirow[t]{2}{*}{ Systems } & \multicolumn{2}{|c|}{ WER [\%] } & \multicolumn{2}{|c|}{ CER [\%] } \\
\hline & Devel & Eval & Devel & Eval \\
\hline GHMM & 31.9 & 38.9 & 8.4 & 11.7 \\
\hline + M-MMI & 25.8 & 31.6 & 7.6 & 11.8 \\
\hline + M-MPE & 24.3 & 30.0 & 6.8 & 10.9 \\
\hline MLP/HMM & 31.2 & 36.9 & 10.0 & 14.2 \\
\hline MLP-GHMM & 25.7 & 32.9 & 7.7 & 12.4 \\
\hline + M-MMI & 23.5 & 30.1 & 6.7 & 11.1 \\
\hline + M-MPE & 22.7 & 28.8 & 6.1 & 10.1 \\
\hline Dreuw et al. [5] (GHMM) & 23.7 & 29.2 & 6.5 & 10.3 \\
\hline Bertolami et al. [1] (GHMM) & 30.9 & 35.5 & - & \\
\hline E. et al. [7] (GHMM) & 32.8 & 38.8 & - & 18.6 \\
\hline Bertolami et al. [1] (HMMs) & 26.8 & 32.8 & - & \\
\hline Graves et al. [8] (RNN/CTC) & - & 25.9 & - & 18.2 \\
\hline E. et al. [7] (MLPs/HMM) & 19.0 & 22.4 & - & 9.8 \\
\hline
\end{tabular}

transferred back to ASR domains. Especially the following conclusions can be drawn:

- retrained tandem MLP-GHMM could always outperform the hybrid MLP/HMM approach (for any considered training criterion, i.e. ML/M-MMI/M-MPE)

- first layer feature reduction in hierarchical MLP networks is uncritical, second layer feature reduction should be about $50 \%$ w.r.t. number of output labels

- the quality of the initial alignment to train the MLP has a high impact on the discriminative quality of the generated log-posterior features

- relative improvements usually achieved with discriminative M-MMI/M-MPE training can also be observed in combination with discriminative MLP features, i.e. effects can be accumulated

- generatively trained systems perform better with more densities, discriminatively trained systems with fewer Interesting for further research will remain hybrid NN / HMM approaches $[7,8,16]$, combining the advantages of large and non-linear context modeling via neural networks while profiting from the Markovian sequence modeling. This is also supported by the $36 \%$ relative improvement we could achieve in the ICFHR 2010 Arabic handwriting competition [14] with the proposed framework.

Acknowledgements. We would like to thank Georg Heigold for his support. This work was partly realized as part of the Google Research Award "Robust Recognition of Machine Printed Text", and as part of the Quaero Programme, funded by OSEO, French State agency for innovation.

\section{REFERENCES}

[1] R. Bertolami and H. Bunke. Hidden Markov model-based ensemble methods for offline handwritten text line recognition.
Pattern Recognition, 41(11):3452-3460, November 2008.

[2] A. E. Biem. Minimum classification error training of hidden Markov models for handwriting recognition. In IEEE ICASSP, volume 3, Salt Lake City, UT, USA, pages 1529-1532, May 2001.

[3] H. Bourland and N. Morgan. Connectionist speech recognition: A hybrid approach. Series in engineering and computer science. Kluwer Academic Publishers, 247, 1994.

[4] P. Dreuw, G. Heigold, and H. Ney. Confidence-Based Discriminative Training for Model Adaptation in Offline Arabic Handwriting Recognition. In ICDAR, Barcelona, Spain, pages 596-600, July 2009.

[5] P. Dreuw, G. Heigold, and H. Ney. Confidence and MarginBased MMI/MPE Discriminative Training for Offline Handwriting Recognition. IJDAR, page accepted, April 2011.

[6] P. Dreuw, S. Jonas, and H. Ney. White-Space Models for Offline Arabic Handwriting Recognition. In ICPR, Tampa, Florida, USA, December 2008.

[7] S. Espana-Boquera, M. Castro-Bleda, J. Gorbe-Moya, and F. Zamora-Martinez. Improving Offline Handwritten Text Recognition with Hybrid HMM/ANN Models. IEEE TPAMI, PP(99):pre-print, 2010.

[8] A. Graves, M. Liwicki, S. Fernandez, R. Bertolami, H. Bunke, and J. Schmidhuber. A Novel Connectionist System for Unconstrained Handwriting Recognition. IEEE TPAMI, 31(5):855-868, May 2009.

[9] G. Heigold, S. Wiesler, M. Nussbaum, P. Lehnen, R. Schlüter, and H. Ney. Discriminative HMMs, Log-Linear Models, and CRFs: What is the Difference? In IEEE ICASSP, Dallas, Texas, USA, pages 5546-5549, March 2010.

[10] G. Heigold, P. Dreuw, S. Hahn, R. Schlüter, and H. Ney. Margin-Based Discriminative Training for String Recognition. IEEE Journal of Selected Topics in Signal Processing - Statistical Learning Methods for Speech and Language Processing, 4(6):917-925, December 2010.

[11] G. Heigold, R. Schlüter, and H. Ney. Modified MPE/MMI in a Transducer-Based Framework. In IEEE ICASSP, Taipei, Taiwan, pages 3749-3752, April 2009.

[12] H. Hermansky and S. Sharma. TRAPs - classifiers of temporal patterns. In ICSLP, 1998.

[13] V. Märgner and H. E. Abed. ICDAR 2009 Arabic Handwriting Recognition Competition. In ICDAR, Barcelona, Spain, pages 1383-1387, July 2009.

[14] V. Märgner and H. E. Abed. ICFHR 2010 Arabic Handwriting Recognition Competition. In ICFHR, November 2010.

[15] U.-V. Marti and H. Bunke. The IAM-database: an English sentence database for offline handwriting recognition. IJDAR, 5(1):39-46, November 2002.

[16] J. Schenk and G. Rigoll. Novel Hybrid NN/HMM Modelling Techniques for On-line Handwriting Recognition. In IWFHR, La Baule, France, October 2006.

[17] F. Valente, J. Vepa, C. Plahl, C. Gollan, H. Hermansky, and R. Schlüter. Hierarchical Neural Networks Feature Extraction for LVCSR system. In Interspeech, Antwerp, Belgium, pages 42-45, August 2007. 